\title{
The story so far
}

\section{Basic research in nanoscience and technology is flourishing, but obstacles to real-world applications remain.}

Five years ago, the editorial in the first issue of Nature Nanotechnology started as follows: "Depending on who you ask, nanotechnology started in 1981, 1974, 1959 or the Bronze Age." That is still true. "And depending on who you believe, and the definitions they use," the article went on, "the world market for nanotechnology products will be worth $\$ 2,600$ billion in 2014 , or $\$ 1,000$ billion in 2015." This remains to be seen, although there are definitely fewer outlandish predictions about the commercial impact of nanotechnology today than there were back in 2006. The editorial continued by pondering some familiar themes that we will return to below - such as the definition of nanotechnology and the need to know more about impact of nanomaterials on the environment and health - and went on to coin a new word to describe the large amount of funding that was flowing into nanoscale science and technology. This author hoped that this word would enter common usage, but half a decade later a Google search returns a meagre three hits for the term 'nanolargesse'.

To mark the first five years of Nature Nanotechnology we have prepared a series of web pages that bring together all the papers we have published in four particularly active areas - DNA nanotechnology, graphene, nanopores and nanotoxicology - along with a collection of articles on the public perceptions of nanotechnology ${ }^{1}$. We could have chosen other areas, but these four seemed to offer the best combination of overall activity and number of relevant papers published in Nature Nanotechnology, and the articles on public perception help place nanotechnology in a wider context.

Research into DNA nanotechnology has branched out in recent years, and in addition to a proliferation of DNA-based nanostructures and devices, this famous molecule is also increasingly being used to organize other nanomaterials (such as nanoparticles and quantum dots). RNA nanotechnology ${ }^{2}$ is also emerging as a growing area.

The paper that kick-started the rise of graphene was published in 2004 (ref. 3), but the number of papers grew only slowly at first, and in its early years Nature
Nanotechnology received many more manuscripts on carbon nanotubes than its two-dimensional cousin. Indeed authors submitted more than 100 papers about nanotubes in 2006, compared with just three on graphene, and nanotubes featured in four of the seven papers published in the first issue. Following the initial gold rush of novel physics, chemists became interested in the production of graphene, and the list of this material's remarkable properties grew longer and longer. However, there remains much to do: the lack of an intrinsic band gap, for example, is still a major disadvantage for applications in electronics ${ }^{4}$.

\section{The number of nanotechnology papers has grown from about 8,000 in 1991 to about 87,000 in 2009.}

The ultimate goal of research into nanopore-based sensors is to be able to sequence the human genome for under $\$ 1,000$. The main advantage of the nanopore approach to sequencing is that it does not require labels or amplification, and workers in the field are developing sensors based on naturally occurring biological nanopores, solid-state nanopores and hybrids of the two. However, each has its own strengths and weaknesses (for example, at present the DNA molecules pass through solid-state nanopores too fast to be sequenced), and nanopore sensors in general face competition from a range of other technologies ${ }^{5}$.

Of course, nanotechnology products will only be able to enter the market if we know for sure that they do not present a threat to health or the environment, and there has been a series of reports over the past five years bemoaning lack of progress in this area. Addressing this problem requires a combination of research in nanotoxicology (including computational approaches) ${ }^{6}$ and changes to the regulations governing the use of chemicals and materials?

Despite what many researchers think, nanotechnology does not get a bad press. Moreover, the public does not fear nanotechnology - indeed the majority know little or nothing about it, and the responses of those who are aware of it depend on a wide range of factors, with some applications being viewed more favourably than others.

Another noticeable trend over the past five years has been the increase in the number of nanotechnology papers (and journals) published. A recent analysis found that the number of nanotechnology papers has grown from about 8,000 in 1991 to about 87,000 in 2009 (ref. 8). Researchers based in China were the most prolific authors, followed by those in the United States, which is not surprising. However, when countries are ranked according to nanotechnology papers as a percentage of all papers, Singapore emerges at the top of the list (16.41\%), followed by China (15.32\%) and South Korea (13.30\%). Nanotechnology accounts for a noticeably smaller percentage of research activity in Japan (8.45\%), the European Union (5.24\%) and the United States $(4.7 \%)$. The complexity of the search query used in this analysis also highlights the difficulties inherent in trying to define nanotechnology.

So what has become of the research published in the first issue of Nature Nanotechnology, and the authors of those papers? One now works for Intel, two have made the jump from graduate student to assistant professor, and the rest are all working as postdoctoral researchers (page 607). Of the senior authors, some still have students and postdocs building on the results they published back in 2006, whereas others have moved into new fields, notably energy-related research and the interface between nanoscience and biology. Based on this (admittedly small) sample, it is also clear that the bulk of progress over the past five years has been in understanding the basic science, rather than developing new technology for realworld applications. Will the same still be true five years from now?

\footnotetext{
References

1. http://www.nature.com/nnano/focus/highlights/index.html

2. Guo, P. Nature Nanotech. 5, 833-842 (2010).

3. Novoselov, K. et al. Science 306, 666-669 (2004).

4. Schwierz, F. Nature Nanotech. 5, 487-496 (2010).

5. Venkatesan, B. M. \& Bashir, R. Nature Nanotech. 6, 615-624 (2011).

6. Nature Nanotech. 6, 329 (2011).

7. Maynard, A. Nature 475, 31 (2011).

8. Grieneisen, M. \& Zhang, M. Small http://dx.doi.org/

10.1002/smll.201100387 (2011)
} 